\title{
Interatrial Septum and Appendage Ostium in Atrial Fibrillation Patients: A Population Study
}

\author{
Anupama Goparaju ${ }^{1}$, Alan Morris ${ }^{2}$, Ibolya $\operatorname{Csecs}^{2}$, Riddhish Bhalodia ${ }^{1}$, Tom Ditter ${ }^{3}$, Kristine \\ Fuimaono $^{3}$, Evgueni Kholmovski ${ }^{4}$, Nassir Marrouche ${ }^{2}$, Joshua Cates ${ }^{2}$, Shireen Elhabian ${ }^{1}$ \\ ${ }^{1}$ Scientific Computing and Imaging Institute, University of Utah, Salt Lake City, UT, USA \\ ${ }^{2}$ Division of Cardiovascular Medicine, School of Medicine, \\ University of Utah, Salt Lake City, UT, USA \\ ${ }^{3}$ Coherex Medical, Salt Lake City, UT, USA \\ ${ }^{4}$ Department of Radiology and Imaging Sciences, School of Medicine, \\ University of Utah, Salt Lake City, UT, USA
}

\begin{abstract}
Left atrial appendage (LAA) closure is performed in atrial fibrillation $(A F)$ patients to help prevent stroke. LAA closure using an occlusion implant is performed under imaging guidance. However, occlusion can be a complicated process due to the highly variable and heterogeneous LAA shapes across patients. Patient-specific implant selection and insertion processes are keys to the success of the procedure, yet subjective in nature. A population study of the angle of entry at the interatrial septum relative to the appendage can assist in both catheter design and patientspecific implant choice. In our population study, we analyzed the inherent clusters of the angles that were obtained between the septum normal and the LAA ostium plane. The number of inherent angle clusters matched the LAA four morphological classifications reported in the literature. Further, our exploratory analysis revealed that the normal from the ostium plane does not intersect the septum in all the samples under study. The insights gained from this study can help assist in making objective decisions during LAA closure.
\end{abstract}

\section{Introduction}

Atrial fibrillation (AF) is a cardiac arrhythmia condition. AF can result in thrombus formation in the LAA and can cause stroke in patients when the thrombus is circulated through the blood. Oral anticoagulation (OAC) therapy is recommended for AF patients to help reduce the risk of stroke [1]. Alternatively, LAA occlusion is proposed for patients with a high risk of stroke [1] because OAC may increase the bleeding risk in patients. LAA closure is often performed using an occlusion implant (see Figure 1),

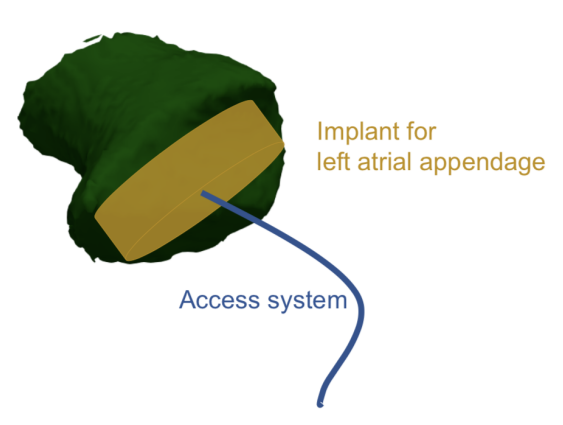

Figure 1. Left atrial appendage closure using an implant and an access system.

which is available in different sizes, through imaging guidance via three-dimensional (3D) transesophageal echocardiography (TEE). The LAA is characterized by its variability in shape and size [2]. Implant size selection based on analyzing the patient-specific LAA is crucial to the success of the LAA closure. The catheter is advanced through the transseptal puncture to allow entry into the LAA. However, the closure process can be worse than no closure in the case of inappropriate implant selection, transseptal puncture, and/or advancement of the catheter to the LAA [3]. Implant catheters are often provided with limited, fixed angles of insertion. Hence, a population-level analysis of the angle of entry at the interatrial septum relative to the appendage can assist in both catheter design and implant choice for a particular patient.

\section{Methods}

The population study was conducted on cardiac MRI images that were retrospectively obtained from an MRI 
image database of AF patients, namely "the AFib database", maintained at the Comprehensive Arrhythmia Research and Management (CARMA) Center at the University of Utah. The MRI images were segmented by an imaging expert with experience segmenting LAA anatomy on MRI. LAA and septum segmentations from MRI were obtained for 127 AF patients. For each patient, we used Corview Software (Marrek Inc., Salt Lake City, UT) to manually annotate LAA ostium landmarks that were then reviewed by a clinical expert. The ostium landmarks (the ostia contour points and center of the ostia) were used to identify the ostium plane using principal component analysis (PCA) (see Figure 2). Similar to the ostium plane, PCA was performed on the points of the septum segmentation to obtain the septum normal. In particular, PCA on septum points or ostium landmarks estimates the lowdimensional subspace, explaining the variability of septum points or the ostium landmarks, respectively. A PCA lowdimensional subspace is parameterized by the eigenvalues and associated eigenvectors. The first two eigenvectors obtained from the PCA on the ostium landmarks define the ostia plane, and the third eigenvector of the PCA on the septum points provides the surface normal to the septum plane. The angle between the ostium plane and septum

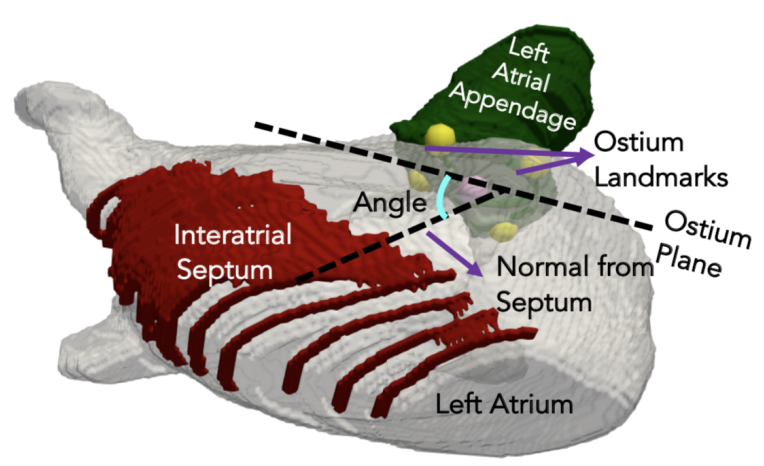

Figure 2. Angle of septum normal relative to ostia plane

normal was computed for all patients. The inherent number of clusters for these angles was identified using the elbow method [4]. The elbow method uses the percentage of variance explained as a function of the number of clusters found in the data. The first few clusters are expected to explain significant variance, but with adding more clusters, the marginal gain in the explained variance drops, resulting in an elbow. Patients' angles and the number of clusters are provided as input to a clustering algorithm (e.g., K-means, $\mathrm{K}$-medoids) to assign cluster labels to patients.

To analyze the spread of LAA types within the clusters of the angles, the LAA shapes, where LAA binary segmentations were converted to signed distance transforms [5], were clustered and classified as cauliflower, chicken wing, wind sock, and cactus [2] with matching characteristics from the literature (see Figure 3). A two-dimensional projection of the clustered LAA shapes from signed distance transform images using t-distributed stochastic neighbor embedding (t-SNE) [6] was obtained to visualize the clusters separability (see Figure 3). We further computed the normal of the ostium plane as the third eigen vector from the PCA of the ostia landmarks and found that this normal was not intersecting the septum segmentation for all the subjects under analysis.

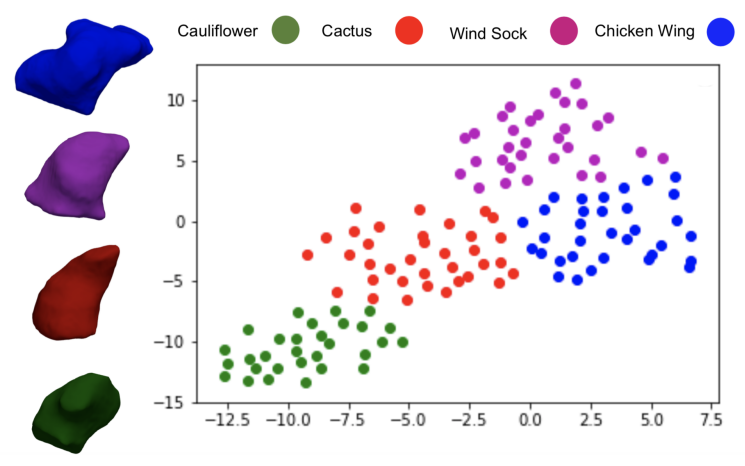

Figure 3. A two-dimensional projection of the clustered LAA shapes from signed distance transform images using $\mathrm{t}$-distributed stochastic neighbor embedding ( $\mathrm{t}-\mathrm{SNE}$ ).

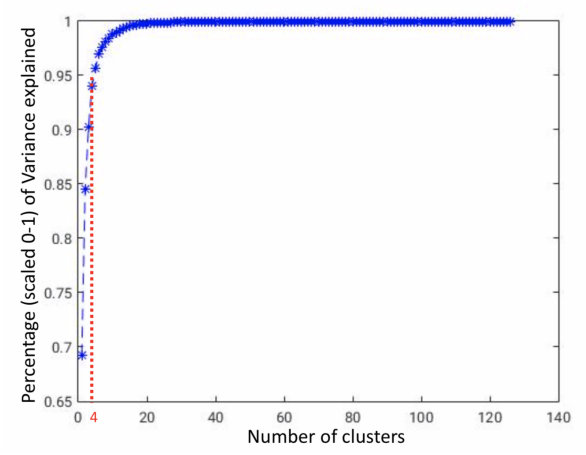

Figure 4. Elbow method on the angles. Four clusters have been found to best explain the angle distribution.

\section{Results}

The angle computation between the normal from the septum and the ostium plane is demonstrated in Figure 2. The elbow method revealed four inherent clusters (see Figure 4). Four angle clusters were found to explain $\sim 90 \%$ of the underlying variability. Angle distributions within each cluster are shown in Figure 5. Angles range from 40 and 160 degrees, with the majority of the angles belonging to the range between 90 and 150 degrees. The angle clus- 
ter counts for each LAA type are listed in Figure 6. LAA data was clustered into four clusters. The representative samples from each cluster were used to visualize the normal from the ostium plane (see Figure 7). The ostium normal is found to not intersect the septum for all four cluster representative samples.

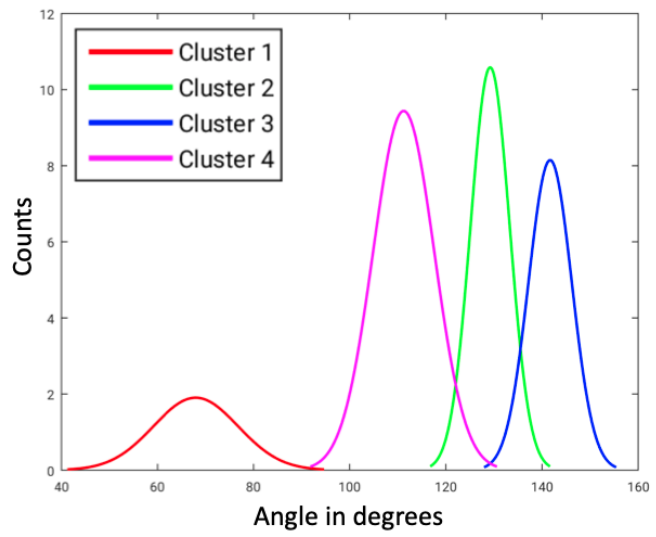

Figure 5. Angle cluster distributions.

\begin{tabular}{|c|c|c|c|c|}
\hline $\begin{array}{l}\text { Angle } \\
\text { Cluster }\end{array}$ & $\begin{array}{l}\text { Cauliflower } \\
\text { LAA }\end{array}$ & $\begin{array}{l}\text { Chicken } \\
\text { Wing LAA }\end{array}$ & $\begin{array}{l}\text { Wind } \\
\text { Sock LAA }\end{array}$ & $\begin{array}{l}\text { Cactus } \\
\text { LAA }\end{array}$ \\
\hline $\begin{array}{l}\text { Cluster } 1 \\
(4 / 127)\end{array}$ & $2 / 4$ & 0 & $2 / 4$ & 0 \\
\hline $\begin{array}{l}\text { Cluster } 2 \\
\text { (58/127) }\end{array}$ & $13 / 58$ & $14 / 58$ & $8 / 58$ & $23 / 58$ \\
\hline $\begin{array}{l}\text { Cluster } 3 \\
\text { (31/127) }\end{array}$ & $6 / 31$ & $2 / 31$ & $8 / 31$ & $15 / 31$ \\
\hline $\begin{array}{l}\text { Cluster } 4 \\
\text { (34/127) }\end{array}$ & $9 / 34$ & $5 / 34$ & $6 / 34$ & $14 / 34$ \\
\hline
\end{tabular}

Figure 6. Angle distributions for LAA clusters.

\section{Conclusion}

Population analysis of the septum normal angle relative to the ostium plane can assist both device manufacturers and clinicians in making decisions for implant catheter design selection. As future work, patient-specific shape modeling will be performed to assist in catheter puncture angle selection and implant device choice.

\section{Acknowledgments}

This work was supported by NIH [grant numbers R01HL135568-02 and P41-GM103545-19] and Coherex Medical.

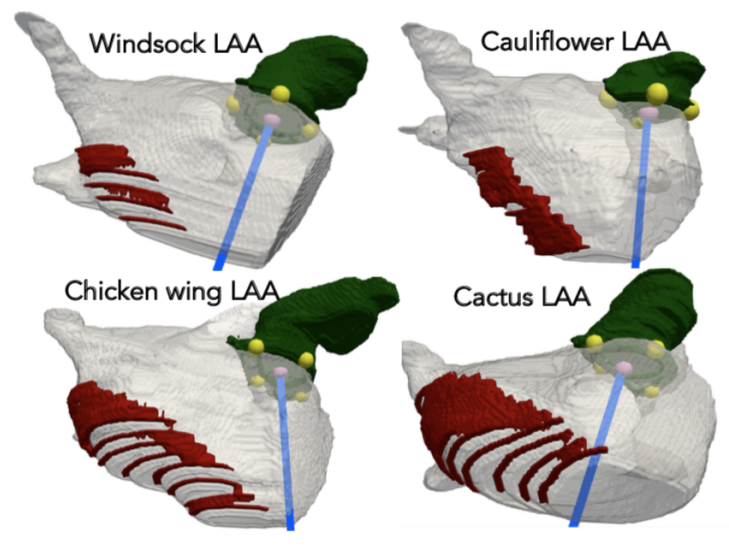

Figure 7. Normal from ostium plane for LAA representative cluster samples.

\section{References}

[1] Fernández AR, González AB. Imaging techniques in percutaneous cardiac structural interventions: atrial septal defect closure and left atrial appendage occlusion. Revista Espanola de Cardiologia English Edition 2016;69(8):766-777.

[2] Wang Y, Di Biase L, Horton RP, Nguyen T, Morhanty P, Natale A. Left atrial appendage studied by computed tomography to help planning for appendage closure device placement. Journal of cardiovascular electrophysiology 2010; 21(9):973-982.

[3] Regazzoli D, Ancona F, Trevisi N, Guarracini F, Radinovic A, Oppizzi M, Marzi A, Sora NC, Della Bella P, Mazzone P, et al. Left atrial appendage: physiology, pathology, and role as a therapeutic target. BioMed research international 2015; 2015.

[4] Hardy A. An examination of procedures for determining the number of clusters in a data set. In New approaches in classification and data analysis. Springer, 1994; 178-185.

[5] Cates J, Elhabian S, Whitaker R. Shapeworks: particle-based shape correspondence and visualization software. In Statistical Shape and Deformation Analysis. Elsevier, 2017; 257298.

[6] Maaten Lvd, Hinton G. Visualizing data using t-sne. Journal of machine learning research 2008;9(Nov):2579-2605.

Address for correspondence:

Shireen Elhabian

Scientific Computing and Imaging Institute, Salt Lake City, Utah, 84112, USA

shireen@sci.utah.edu 